This document is the Accepted Manuscript version of a Published Work that appeared in final form in Journal of the American Chemical Society, copyright (c) American Chemical Society after peer review and technical editing by the publisher. To access the final edited and published work see:

https://dx.doi.org/10.1021/jacs. 9b12389. 


\section{Biomimetic synthesis of sub-20 nanometer Covalent Organic Frameworks in water}

\section{Authors}

Carlos Franco ${ }^{1} \uparrow$, David Rodríguez-San-Miguel ${ }^{2} \uparrow$, Alessandro Sorrenti ${ }^{1}$, Semih Sevim ${ }^{1}$, Ramon Pons $^{3}$, Ana E. Platero-Prats ${ }^{2}$, Marko Pavlovic ${ }^{4,5}$, Istvan Szilágyi ${ }^{4,6}$, M. Luisa Ruiz Gonzalez ${ }^{7}$, José M. González-Calbet ${ }^{7}$, Davide Bochicchio ${ }^{8}$, Luca Pesce $^{8}$, Giovanni M. Pavan ${ }^{8}$, Inhar Imaz ${ }^{9}$, Mary Cano-Sarabia $^{9}$, Daniel Maspoch ${ }^{9,10}$, Salvador Pané ${ }^{11}$, Andrew J. deMello ${ }^{1}$, Felix. Zamora ${ }^{2 *}$, Josep Puigmartí-Luis ${ }^{1 *}$

\section{Affiliations}

${ }^{1}$ Department of Chemistry and Applied Biosciences, Institute for Chemical and Bioengineering, ETH Zurich, Vladimir Prelog Weg 1, 8093 Zurich, Switzerland.

${ }^{2}$ Departamento de Química Inorgánica, Institute for Advanced Research in Chemical Sciences (IAdChem) and Condensed Matter Physics Institute (IFIMAC). Universidad Autónoma de Madrid, 28049 Madrid, Spain.

${ }^{3}$ Institute for Advanced Chemistry of Catalonia (IQAC-CSIC), Jordi Girona 18-26, E-08034 Barcelona, Spain.

${ }^{4}$ MTA-SZTE Lendület Biocolloids Research Group, University of Szeged, H-6720 Szeged, Hungary.

${ }^{5}$ Max-Planck Institute of Colloids and Interfaces; Department of Colloid Chemistry, Am Mühlenberg 1, 14476 Potsdam, Germany

${ }^{6}$ Interdisciplinary Excellence Center, Department of Physical Chemistry and Materials Science, University of Szeged, H-6720 Szeged, Hungary.

${ }^{7}$ Departamento de Química Inorgánica, Universidad Complutense de Madrid, 28040 Madrid, Spain.

${ }^{8}$ Department of Innovative Technologies, University of Applied Sciences and Arts of Southern Switzerland, Galleria 2, Via Cantonale 2c, CH-6928 Manno, Switzerland.

${ }^{9}$ Catalan Institute of Nanoscience and Nanotechnology (ICN2), CSIC and BIST Campus UAB, Bellaterra, 08193 Barcelona, Spain.

${ }^{10}$ ICREA, Pg. Lluís Companys 23, 08010 Barcelona, Spain.

${ }^{11}$ Multi-Scale Robotics Lab ETH Zurich Tannenstrasse 3, CH-8092 Zurich, Switzerland.

*Correspondence to: felix.zamora@uam.es, jpuigmarti@ethz.ch

$\uparrow$ These authors contributed equally to this work. 


\section{Abstract}

38 Covalent organic frameworks (COFs) are commonly synthetized under harsh conditions yielding unprocessable powders. Control in their crystallization process and growth has been limited to

40 studies conducted in hazardous organic solvents. Herein, we report a one-pot synthetic method that

41 yields stable aqueous colloidal solutions of sub-20 nm crystalline imine-based COF particles at

42 room temperature and ambient pressure. Additionally, through the combination of experimental

43 and computational studies, we investigated the mechanisms and forces underlying the formation of

44 such imine-based COF colloids in water. Further, we show that our method can be used to process

45 the colloidal solution into 2D and 3D COF shapes, as well as to generate a COF ink that can be

46 directly printed onto surfaces. These findings should open new vistas in COF chemistry enabling

47 new application areas.

\section{Introduction}

50 Covalent organic frameworks (COFs) are porous crystalline materials generated from organic

51 molecules linked via reversible covalent bonds. ${ }^{1}$ Since its discovery, COF chemistry has facilitated

52 a modular construction of periodic crystalline matter by connecting molecular subunits in a

53 predictable and modular fashion. ${ }^{2}$ This strategy has proved efficient in generating extended

54 crystalline and porous networks possessing permanent porosity, high specific surface areas and

55 excellent thermal/chemical stability; features that have found potential applications in a vast

56 number of fields. ${ }^{3}$ However, conventional routes for COF synthesis involve high temperatures,

57 which when combined with the low solubility of the initial building blocks in common reaction

58 media, yield poor control over the size of the crystalline domains and the morphology of COF

59 crystals. $^{4}$ Unsurprisingly, such drawbacks have hampered the extraction of reliable information

60 regarding the effects of crystallite size and morphology on COF properties. Accordingly, much 
61 effort is now focused on both understanding and controlling the growth of COF crystals at length

62 scales spanning the nanometer to micron scales.

63 Recently, Dichtel and co-workers reported on the preparation of stable particles of boronate ester-

64 linked COFs, whose size can be modulated between 40 and hundreds of nanometers by using

65 mixtures of organic solvents at high temperature. ${ }^{5}$ Later, such COFs colloidal solutions in organic

66 media have been used by the same authors for preparing micron-sized single crystals of boronate

67 ester-linked COFs via a seeded growth procedure. ${ }^{6}$ Therefore, having access to nanometer-sized

68 particles of COFs allowed the authors to overcome a long-standing challenge in the field, i.e. the

69 formation of large single crystals of COFs. Besides this specific example, COF crystal-downsizing

70 will be key to transforming COFs from unprocessable crystalline powders into processable

71 materials, integrating COFs into nanoscale devices, ${ }^{7}$ as well as establishing relationships between

72 COF crystal size and properties. In addition, COF crystal downsizing will expand the range of

73 applications of these materials, such as in the biomedical, device and printing arenas, ${ }^{8}$ and enhance

74 their bioavailability. ${ }^{9}$ However, only nanoparticles of boronate ester-linked COFs in organic

75 solvents have been reported so far. ${ }^{5}$ Unfortunately, boron-based COFs have poor chemical

76 stabilities, which limits their practical implementation. In addition, the fact that hazardous organic

77 solvents are still required as a medium to stabilize their colloidal dispersion precludes their use in

78 biological environments. In contrast, imine-based COFs are significantly more stable and robust

79 for practical use. ${ }^{10}$ Nevertheless, despite the high number of reports on imine-based COFs, it has

80 not been possible yet to downsize them to the nanoscale. ${ }^{11}$ Put simply, routes for producing aqueous

81 colloidal solutions of imine-linked COF nanoparticles are still missing.

82 To overcome this limitation, we report here an efficient one-pot method to generate stable and

83 homogeneous colloidal solutions of sub-20 nanometer imine-linked COF particles in water. The 
84 synthesis of crystalline COF colloids was performed for the first time at room temperature using

85 micelles as reaction nanocompartments. This approach was inspired by living systems that make use of confined volumes (such as intracellular compartments) to control crystallization processes

87 in aqueous media. ${ }^{12-14}$ This method allowed us to use a combination of experimental scattering techniques in solution, that together with computational calculations, gave unprecedented insights into the mechanism and forces underlying the formation of imine-linked COFs. Additionally, we show that the produced colloids enable the processing of COFs into $2 \mathrm{D}$ and $3 \mathrm{D}$ shapes such as crystalline freestanding films and monoliths. Further, we prove that such colloids can also be used

92 as inks to directly print COFs onto surfaces. Finally, we demonstrated the generality of our method

93 by applying it to the synthesis of metal-organic frameworks (MOFs) colloids. In particular, we 94 show the synthesis of 20 nanometer MIL-100(Fe) particles at room temperature and ambient pressure. MIL-100(Fe) is a prototypical MOF that requires harsh conditions for its synthesis ${ }^{15}$ and only forms larger crystals. ${ }^{16}$ We expect that the presented methodology will vastly increase knowledge on structure-property correlations in COFs and MOFs, allowing access to a large number of new applications and functions, whilst significantly enhancing the bioavailability and 99 processability of these materials.

\section{Results}

101 TAPB-BTCA COF is typically obtained via imine condensation between 1,3,5-tris(4102 aminophenyl)benzene (TAPB) and 1,3,5-triformylbenzene (BTCA) in meta-cresol or DMSO. 103 Additionally, acetic acid is used as a catalyst to yield TAPB-BTCA COF as an insoluble and unprocessable crystalline powder. ${ }^{17}$ Herein, we employed the catanionic micellar system ${ }^{18,19}$ 105 formed from a mixture of cationic hexadecyltrimethylammonium bromide (CTAB) and anionic sodium dodecyl sulfate (SDS) surfactants (CTAB/SDS 97:3) to generate stable colloidal solutions 
of crystalline TAPB-BTCA COF nanoparticles in water (Fig. 1A). Note that this surfactant ratio

108 guarantees the formation of small mixed micelles in the catanionic mixture, instead of bigger vesicles as previously reported, ${ }^{18}$ and here it was optimized to achieve the smallest size of TAPB-

110 BTCA COF nanoparticles (Fig. S1). The micellar medium allows the solubilization in water of

111 the otherwise insoluble molecular building blocks BTCA and TAPB at room temperature, yielding

112 two homogeneous solutions of the reactants loaded into CTAB/SDS mixed micelles. ${ }^{20}$ After 113 mixing the solutions and adding acetic acid, the reaction mixture turned orange, indicating the 114 formation of imine bonds characteristic of TAPB-BTCA COF growth. However, and in contrast 115 to observations in standard synthetic protocols, the reaction mixture remained clear and 116 homogeneous with no apparent precipitation (Fig. 1B), even after storage at room temperature for 117 twelve months (Fig. S3). Indeed, when irradiated with a laser $(\lambda=630 \mathrm{~nm})$, the reaction mixture 118 clearly exhibited Willis-Tyndall scattering behavior, ${ }^{21}$ confirming the presence of colloidal 119 particles (Fig. S2). To validate the existence of crystalline TAPB-BTCA COF nanoparticles in the 120 reaction mixture, synchrotron X-ray diffraction measurements were performed directly on the colloidal solution generated after mixing. The experimental differential diffraction data were fitted 122 using the Le Bail method ${ }^{22,23}$ against the reported structural model for TAPB-BTCA COF (P3, a $123 \sim 15.91 \AA$ and $\mathrm{c} \sim 3.54 \AA$ as refined cell parameters) (Fig. 1C), demonstrating the presence of the 124 crystalline $\mathrm{COF}$ phase with a main low-angle peak centered at $0.86^{\circ} 2$-theta values $(\lambda=0.2068 \AA)$ 125 associated with the (100) Bragg reflection. ${ }^{17}$ Accordingly, this result unambiguously confirmed the 126 formation of crystalline TAPB-BTCA COF nanoparticles via the mixed micelle method. The sizes and morphology of the obtained TAPB-BTCA COF nanoparticles were subsequently studied by dynamic light scattering (DLS) and cryogenic transmission electron microscopy (cryo-TEM). DLS 129 measurements conducted on the reaction mixture after 24 hours (Fig. S3A) reported a 130 monodisperse distribution of scatterers centred at $16 \mathrm{~nm}$. Remarkably, the colloidal behaviour of 
131 the reaction mixture remains stable and homogeneous (with no appreciable turbidity or size

132 increase) for periods in excess of six months (Fig. S3B). Additionally, cryo-TEM images of the

133 reaction mixture after 24 hours (Fig. 1D) showed two different populations of objects; one centred

134 at $5 \pm 1 \mathrm{~nm}$ and the other at $16 \pm 1 \mathrm{~nm}$ in diameter. The former value correlated well with the size of

135 surfactant micelles determined by small-angle X-ray scattering (SAXS) in the pure CTAB/SDS

136 (97:3) mixture (see below and Fig. S11), with the latter comparing well with the size distribution

137 measured by DLS, and thus being ascribed to TAPB-BTCA COF nanoparticles. The high-

138 resolution transmission electron microscopy (HR-TEM) study of drop cast reaction mixtures

139 further confirmed the crystallinity of TAPB-BTCA COF nanoparticles. Fig. 1E shows a

140 characteristic HRTEM image and its corresponding Fast Fourier Transform (FFT). The measured

141 periodicities (white arrows in Fig. 1E) match well with the unit cell geometry of TAPB-BTCA

142 COF as viewed along the [-211] zone axis. Fig. 1F presents a magnified detail of the above HR-

143 TEM image overlapped with the simulated crystal structure of TAPB-BTCA COF viewed along

144 the [-211] zone axis, suggesting a good match between the light and dark fringes of the micrograph

145 and the higher and lower atomic density regions of the COF structure. Additionally, scanning

146 electron microscopy (SEM) images of drop cast reaction mixtures revealed the presence of well-

147 defined and uniform nanoparticles (and nanoparticle clusters), with a size that correlates well with

148 both DLS and cryo-TEM measurements (Fig. S1A). 

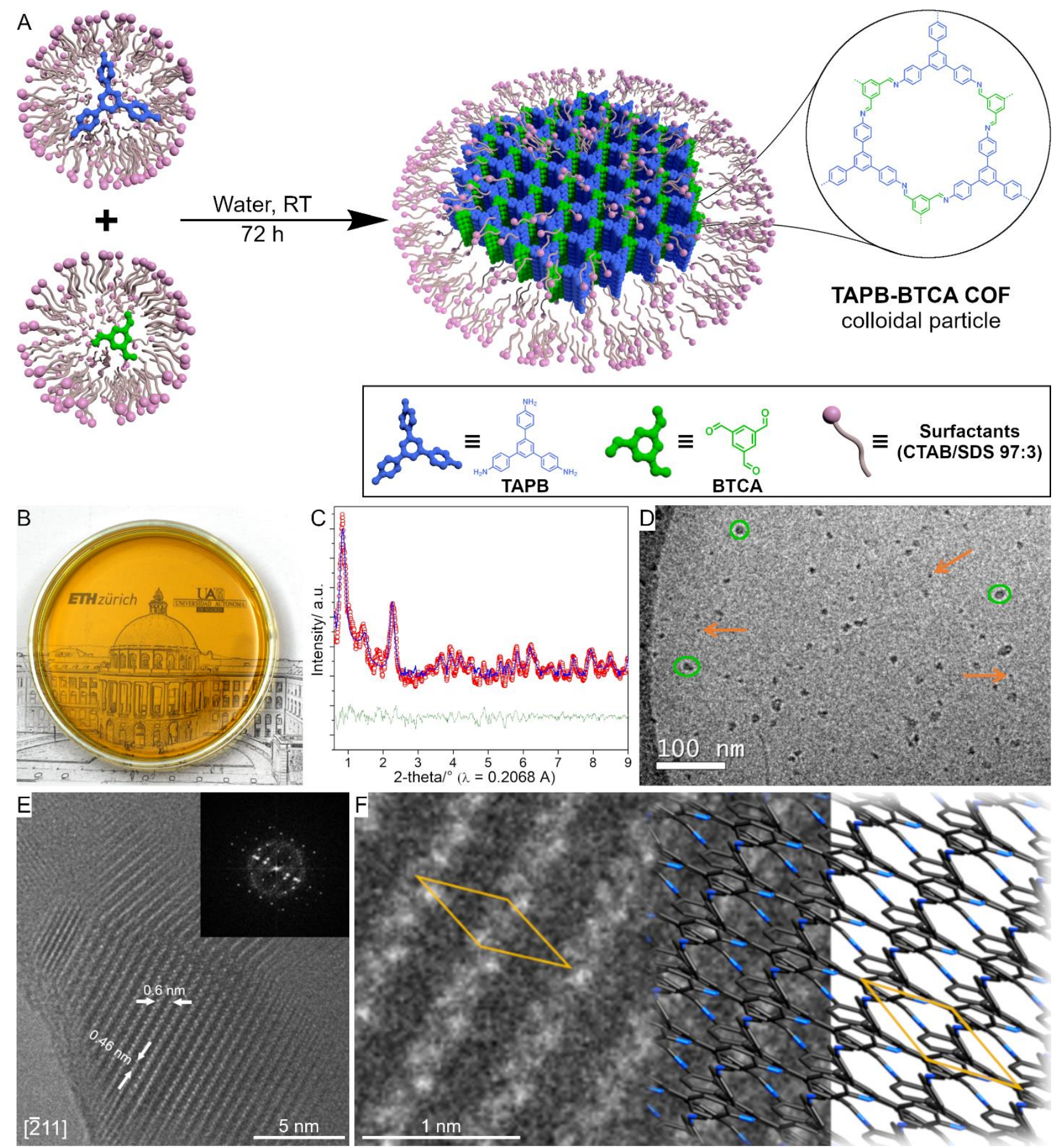

Fig. 1. TAPB-BTCA COF nanoparticles. (A) Schematic representation of the synthesis of colloidal TAPB-BTCA

COF nanoparticles in water. (B) Photograph of the transparent reaction mixture. (C) Synchrotron X-ray diffraction

pattern of the reaction mixture containing TAPB-BTCA COF nanoparticles. Experimental differential data are shown

in red, with the calculated fit using $P 3, \mathrm{a} \sim 15.91 \AA$ and $\mathrm{c} \sim 3.54 \AA$ as refined cell parameters in blue and associated 
BTCA COF nanoparticle along the [-211] zone axis, with the inset showing the FFT. (F) Magnified HR-TEM image of a defined area in (E) overlaid with the schematic structural model of TAPB-BTCA COF along the [-211] projection.

After confirming that sub-20 nanometer TAPB-BTCA COF particles can be generated, we investigated the possibility of isolating the COF material as a bulk solid. To this purpose, , we added ethanol to the reaction mixture to destabilize the surfactant aggregates, ${ }^{24}$ which triggered the flocculation of TAPB-BTCA COF nanoparticles as an insoluble yellow powder, hereafter termed TAPB-BTCA COF(s). After flocculation, TAPB-BTCA COF(s) could be simply isolated from the reaction mixture by centrifugation. TAPB-BTCA COF(s) was characterized by Fouriertransform infrared (FT-IR) spectroscopy and solid-state Cross Polarization/Magic Angle Spinning Nuclear Magnetic Resonance $\left({ }^{13} \mathrm{C}\right.$ CP-MAS NMR). FT-IR spectra confirmed the presence of imine bonds through the appearance of the characteristic imine $\mathrm{C}=\mathrm{N}$ stretching band at $1623 \mathrm{~cm}^{-1}$ (Fig. S4), while solid state ${ }^{13} \mathrm{C}$ CP-MAS NMR spectra exhibited the representative signal of the imine carbon atom at 157.1 ppm (Fig. S5). Additionally, powder X-ray diffraction (PXRD) patterns of TAPB-BTCA COF(s) (Fig. S6) were in excellent accordance with those previously reported for this material. ${ }^{17}$ It should be noted that the measured PXRD peaks were broader than those usually 172 observed for TAPB-BTCA COF(s) prepared by conventional bulk synthetic methods, ${ }^{11}$ 173 suggesting the presence of smaller crystalline domains in TAPB-BTCA COF(s). ${ }^{25}$ The permanent 174 porosity of TAPB-BTCA $\mathbf{C O F}(\mathbf{s})$ was also confirmed by nitrogen adsorption isotherm measurements on previously activated samples, showing a characteristic isotherm with a Brunauer-

176 Emmet-Teller $(\mathrm{BET})$ area $\left(A_{B E T}\right)$ of $687 \mathrm{~m}^{2} \mathrm{~g}^{-1}$ at $77 \mathrm{~K}$ (Fig. S7). Finally, the $\mathrm{CO}_{2}$ and water 177 sorption properties of TAPB-BTCA COF(s) were also measured (Fig. S8 and S9). It was found 178 to be porous to $\mathrm{CO}_{2}$ with a total uptake of $9 \mathrm{mmol} \mathrm{g}-1$ at $203 \mathrm{~K}$ and 760 torr $\left(1 \mathrm{mmol} \mathrm{g}^{-1}\right.$ at $298 \mathrm{~K}$ 179 and 760 torr). Moreover, water-vapour sorption isotherms showed a step between 40-50\% relative 
humidity, after which the water uptake increases monotonically until a maximum of $15 \%$ in mass

$181\left(0.15 \mathrm{~g}_{\text {water }} \mathrm{g}_{\mathrm{COF}}{ }^{-1}\right)$, which is the typical behaviour for this class of materials bearing hydrophobic 182 walls. $^{11}$

183 To clarify the processes underlying the formation of TAPB-BTCA COF nanoparticles in the 184 catanionic micellar medium, time-resolved in-situ DLS and SAXS experiments were performed.

185 DLS indicated that the average hydrodynamic diameter of colloidal particles increased during the 186 first few hours (after the addition of acetic acid), leveling off to yield a final average hydrodynamic 187 diameter of $16 \mathrm{~nm}$ (Fig. S10). In contrast, when the synthesis was performed in pure CTAB 188 micelles (i.e. without SDS), the size of TAPB-BTCA COF continued to increase until precipitation occurred. Accordingly, the role of the anionic surfactant was clearly evidenced, with SDS reducing the electrostatic repulsion of CTAB heads in the micellar aggregates (i.e. decreasing the surface energy), and favoring the formation of assemblies with lower curvatures. ${ }^{19,26}$ This in turn, 192 facilitated the colloidal stabilization of COF oligomers and the final TAPB-BTCA COF 193 nanoparticles by the CTAB/SDS mixed surfactant system, even over extended periods of time. 194 Time-resolved SAXS experiments provided further insights into the growth mechanism of TAPB195 BTCA COF nanoparticles. SAXS spectra of the two micellar solutions containing the TAPB and BTCA precursors (in the presence of acetic acid) indicated the existence of $4.8 \pm 2 \mathrm{~nm}$ diameter spherical micelles; comparable to what it was observed in pure CTAB/SDS (97:3) solutions (Fig. S11). These data indicate that solubilization of COF precursors has a negligible effect on the size and shape of the CTAB/SDS micellar aggregates. However, after mixing the two micellar solutions

200 loaded with COF precursors, clear changes in the SAXS profiles were observed as a function of 201 time. Scattering profiles at selected reaction times (5, 13, and 21 hours) are shown in Fig. 2, along 202 with their best fits obtained from the used scattering model (further details are provided in 
203 Supplementary Materials). These three SAXS spectra describe three different regimes during the 204 progress of the reaction (Fig. 2A-C and Fig. S12). At short reaction times (5 hours in Fig. 2A), 205 SAXS profiles fit well to a disk-particle model with a radius of $6.4 \mathrm{~nm}$ and a thickness of 0.354 206 nm, which corresponds to a single layer of bare TAPB-BTCA COF (Fig. S13A and Table S1). 207 As the reaction proceeded (13 hours in Fig. 2B), SAXS data showed a significant increase in 208 intensity at low values of the scattering vector $\left(\mathrm{q}<1 \mathrm{~nm}^{-1}\right)$, together with the appearance of a broad 209 feature around $2 \mathrm{~nm}^{-1}$, suggesting changes of electron density contrast (further discussion is 210 provided in the Supplementary Materials). ${ }^{27}$ This spectrum could then be better fitted to a COF211 core@double-shell disk model, with a core thickness of $0.91 \mathrm{~nm}$ corresponding to a three-layered TAPB-BTCA COF stack surrounded by surfactant molecules (Fig. S13B and Table S1). At longer 213 reaction times (21 hours in Fig. 2C), the SAXS profile showed a marked change at $\mathrm{q}<1 \mathrm{~nm}^{-1}$, with 214 a clear slope variation at $0.5 \mathrm{~nm}^{-1}$. This spectrum could also be described using a COF215 core@double-shell disk model, but with a core radius of $11 \mathrm{~nm}$ and a thickness of $3.74 \mathrm{~nm}$ (Fig. 216 S13B and Table S1). This thickness corresponds to ten-layered TAPB-BTCA COF stacks fully 217 covered by surfactant. Importantly, these extracted values were in good agreement with the overall 218 size of the colloidal particles as measured by DLS and cryo-TEM. It should be noted that the 219 formation of a compact surfactant layer around the COF nanoparticles is crucial for their 220 stabilization in the reaction mixture, preventing further growth and flocculation. Accordingly, 221 SAXS data suggest that after an initial phase of lateral growth by covalent polymerization, the 222 increase in size of TAPB-BTCA COF nanoparticles is essentially driven by the $\pi-\pi$ stacking of 223 COF layers (Table S1). Coarse grained molecular dynamics (CG-MD) simulations of single-, 224 three- and ten-layered TAPB-BTCA COF particles were performed to gain further insight into the 225 forces driving the self-assembly process. The simulations were run in water as well as hexadecane 

further details about the CG model and simulations).

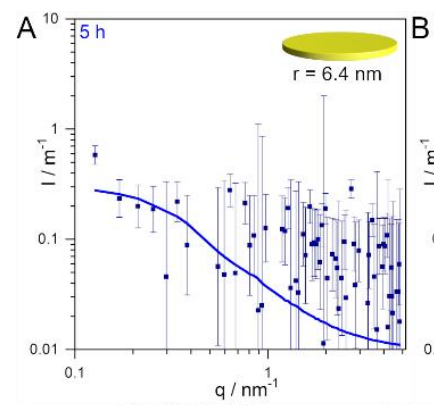

$\mathrm{D}$
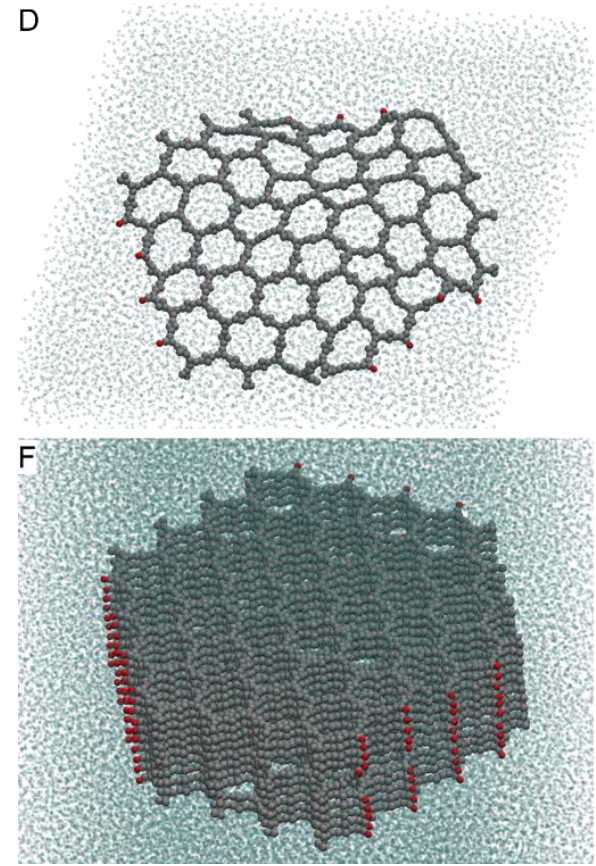

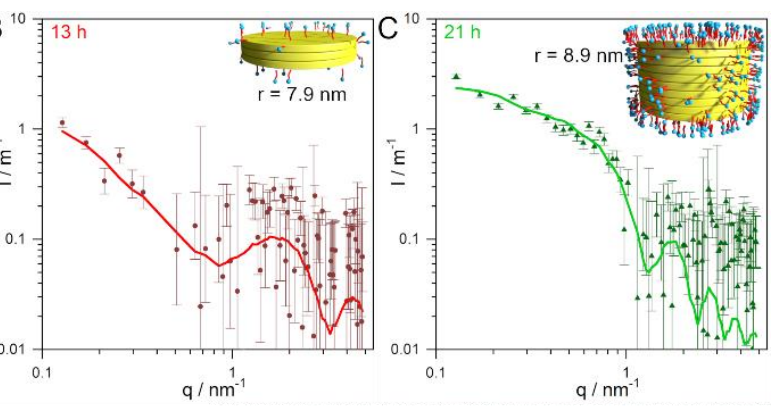

$\mathrm{E}$
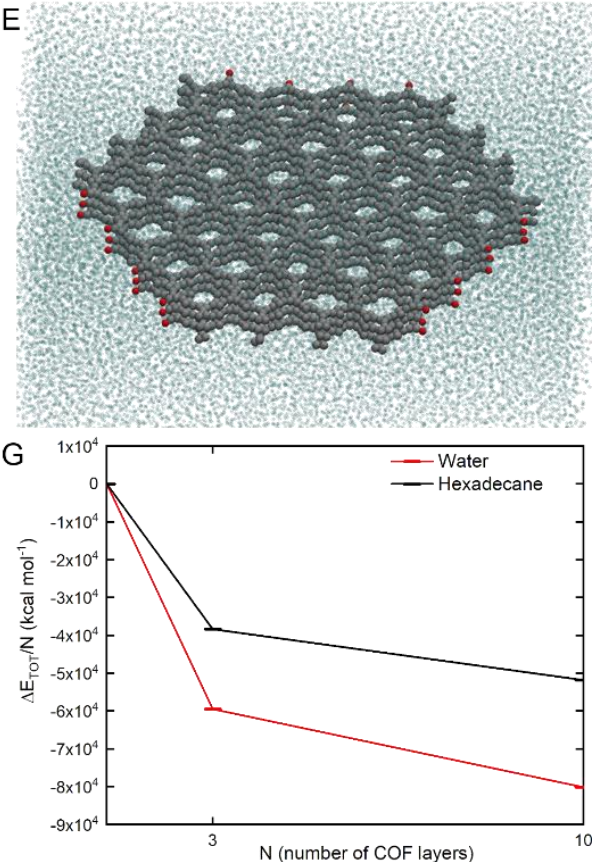

229 Fig. 2. Growth of TAPB-BTCA COF nanoparticles. (A), (B) and (C) SAXS spectra of the reaction mixture at 5, 13 and 21 hours, respectively. Experimental data (symbols) and best fits to the used scattering model (line). The insets illustrate the species measured at the three different regimes, with yellow disks representing the TAPB-BTCA COF core, red cylinders the hydrophobic tails of the surfactants, and blue spheres their polar heads. (D), (E) and (F) Snapshots of TAPB-BTCA COF assemblies comprising 1, 3 and 10 layers respectively, after CG-MD simulation in water. (G) Total energy for the simulations of TAPB-BTCA COF assemblies in water and hexadecane. Energies are normalized by dividing the total energy by the number of layers, $N$. 
237 Fig. 2 D-F present snapshots of the equilibrated assemblies in water (see Fig. S14 for the associated 238 simulations in hexadecane). Simulations confirmed the strong cooperativity in the interaction of 239 the COF layers in both solvents, with a significant reduction in the normalized energy per-layer for 240 the three and ten-layered TAPB-BTCA COF particles when compared to the isolated monolayer 241 (Fig. S15). The calculated energy of stabilization was similar in both solvents (Fig. S15). This 242 suggests that there is a strong preference for COF layers to aggregate, even within the hydrophobic 243 environment, due to a direct enthalpic contribution. However, when considering the total energy 244 of the simulated system (i.e. including the solvent contribution), there is a clear difference between 245 the behavior of the system in water or hexadecane (Fig. 2G). Specifically, COF layers are more 246 likely to aggregate in water (higher cooperativity), suggesting that in this case the driving force for 247 aggregation predominantly arises due to an increase in the water-water interactions upon COF 248 aggregation; a signature of the hydrophobic effect. In addition, MD simulations reveal a higher 249 flexibility of the COF single-layer, which deformed significantly during the simulations (e.g. Fig. 250 2D and Fig. S14) compared to the stacked systems (Fig. 2E-F). These data also explain the greater 251 tendency of surfactant molecules to interact with thicker assemblies (as measured by SAXS), since 252 rigid COF stacks have more extended hydrophobic patches (e.g. pore walls) than rippled single253 layers.

254 In addition to the importance of obtaining colloidal solutions of sub-20 nanometer COF particles 255 in water, the described methodology also offers new opportunities for particle processing. Indeed, 256 until now, a major limitation for the further implementation of COFs outside of laboratory 257 environments has been their unprocessable nature. ${ }^{28}$ Here, we show that by controlling the 258 flocculation and aggregation of TAPB-BTCA COF nanoparticles in the reaction mixture (through 259 the addition of ethanol), 2D and 3D TAPB-BTCA COF shapes could be easily achieved. For 
example, films of TAPB-BTCA COF(s) on the millimeter scale were prepared by confining a

261 concentrated reaction mixture into a home-made microengineered clamp (Fig. 3A), followed by

262 evaporation of the solvent. The concentrated reaction mixture was prepared by exchanging water

263 for ethanol (further details are provided in the Supplementary Materials). We observed that highly

264 uniform freestanding films with thicknesses of approximately $500 \pm 10 \mathrm{~nm}$ were efficiently

265 obtained via this approach (Fig. 3B). Alternatively, reducing the size of the home-made

266 microengineered clamp to squares of $500 \mu \mathrm{m}$ lateral size or even changing its $2 \mathrm{D}$ shape to $3 \mathrm{D}$

267 morphologies led to the generation of smaller TAPB-BTCA COF(s) films (Fig. S16) or 3D

268 octahedrons (Fig. 3C and Supplementary Materials). SEM analysis of these structures showed a

269 nanoparticulated texture similar to the one observed for TAPB-BTCA COF(s) (Fig. S17). These

270 data indicate that the processing steps allowed TAPB-BTCA COF(s) to be shaped into 2D and 3D

271 morphologies, with negligible reductions in the integrity of the COF material. Additionally, PXRD

272 patterns of these structures were identical to those previously reported in the literature for this COF

273 (Fig. S18A). Interestingly, the controlled diffusion of ethanol to the reaction mixture through a 3D

274 flow-focusing microfluidic device allowed us to generate a processable COF ink from the initial

275 colloidal solution. Indeed, the laminar flow conditions operating within such a device provided

276 control over the flocculation and aggregation of TAPB-BTCA COF nanoparticles (Fig. 3D).

277 Accordingly, a direct printing of TAPB-BTCA COF(s) onto surfaces was possible through the

278 tubing connected to the outlet of the microfluidic device (Fig. 3E and 3F and Video S1). PXRD

279 analysis of the printed structures confirmed that TAPB-BTCA COF(s) was deposited (Fig. S18B). 

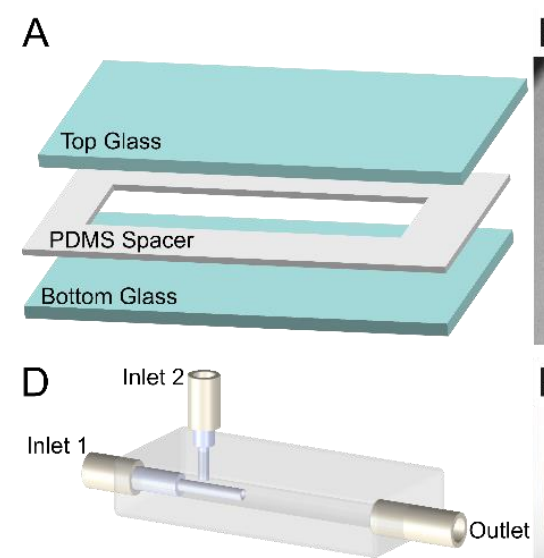

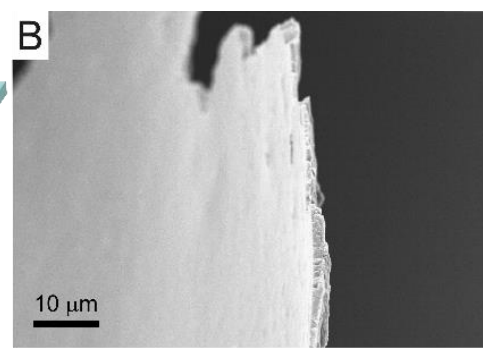

E

$\underline{10 \mathrm{~mm}}$

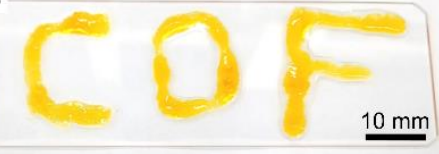

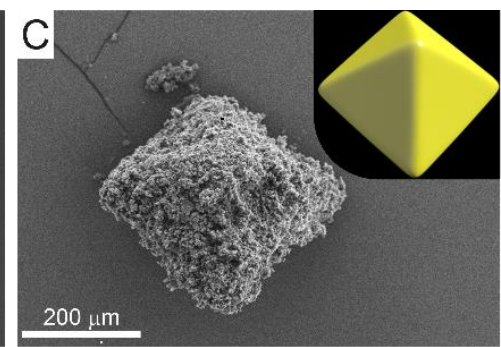

$\mathrm{F}$

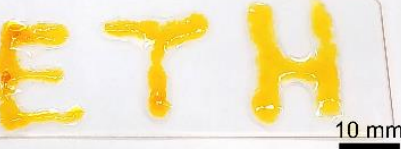

Fig. 3. Processability of the reaction mixture. (A) Schematic illustration of the home-made microengineered clamp used to generate TAPB-BTCA COF(s) films. (B) SEM image of the cross-section of a freestanding mm-sized film obtained using the setup shown in (A). (C) SEM image of a TAPB-BTCA COF(s) octahedron (500 $\mu \mathrm{m}$ edge). (D) Schematic illustration of the continuous 3D flow-focusing microfluidic device used to print TAPB-BTCA COF(s).

The reaction mixture was directly injected through inlet 1, while ethanol was introduced via inlet 2. (E) and (F) Photographs of "COF" and "ETH" printed with TAPB-BTCA COF on a planar surface using the device shown in (D).

To demonstrate the generality of our method, we prepared another imine-based COF, namely TzCOF ${ }^{29}$ via the reaction of 2,4,6-tris(4-aminophenyl)-1,3,5-triazine and BTCA in a CTAB/SDS (97:3) mixture. SEM, DLS, and PXRD analysis clearly confirmed the formation of Tz-COF particles with a size distribution centered around $20 \mathrm{~nm}$ (Fig. S19-S22). ${ }^{30}$ Permanent porosity was measured using BET analysis, with results agreeing with previously reported values for the same COF material (Fig. S23). ${ }^{31}$ Finally, it is significant to note that our method can be extended to MOFs. To demonstrate such generality, we synthesized a prototypical MOF that requires harsh conditions to crystalize, i.e. MIL-100(Fe $)^{15}$. In-situ synchrotron X-ray diffraction measurements of the homogenous reaction mixture clearly confirmed the formation of MIL-100(Fe) (Fig. S24). Furthermore, DLS measurements of drop-cast reaction mixtures indicated a particle size distribution centered around $20 \mathrm{~nm}$ (Fig. S25). To the best of our knowledge, this is the smallest size reported for this biodegradable and non-toxic MOF. ${ }^{32}$ After flocculation of the colloid with 
302 ethanol, PXRD and BET analysis of the resulting powder additionally confirmed the formation of

303 MIL-100(Fe), (Fig. S26 and Fig. S27, respectively). Surprisingly, and in spite of the nanometer 304 size of the generated MIL-100(Fe) particles, the measured BET surface area was high $\left(1068 \mathrm{~m}^{2} \mathrm{~g}^{-}\right.$ $\left.305^{1}\right)$.

\section{Discussion}

307 In summary, we have demonstrated a mild procedure for the preparation of stable aqueous colloidal 308 solutions of crystalline imine-linked COF nanoparticles assisted by micelles of a catanionic 309 surfactant mixture. The micellar medium provides control over the growth of the COF crystallites, 310 which allowed us to reach the smallest size for COF particles among those reported so far. 311 Additionally, by a combination of experimental and computational studies, we were able to shed 312 light into the mechanism and forces underlying the growth of such COF colloids. Note that this 313 mechanistic study is unprecedented for imine-based COFs. Remarkably, the colloidal nature of the 314 formed imine-based COF nanoparticles enabled their processing into 2D and 3D shapes, as well as 315 the generation of an ink for their direct printing onto surfaces. Finally, to demonstrate the generality 316 of our method we extended it to the preparation of colloidal nanoparticles of other porous 317 crystalline materials, such as MOFs. We foresee that the preparation of chemically stable and easily 318 processable imine-based COF colloids will open the door to new applications of these materials, 319 for example in the field of functional devices, due to improved integration possibilities, or 320 biomedicine, thanks to improved bioavailability. 


\section{References}

323

324

325

326
(1) Côté, A. P.; Benin, A. I.; Ockwig, N. W.; O’Keeffe, M.; Matzger, A. J.; Yaghi, O. M. Porous, Crystalline, Covalent Organic Frameworks. Science 2005, 310 (5751), 1166-1170. https://doi.org/10.1126/science.1120411.

(2) Feng, X.; Ding, X.; Jiang, D. Covalent Organic Frameworks. Chemical Society Reviews 2012, 41 (18), 6010-6022. https://doi.org/10.1039/C2CS35157A.

(3) L. Segura, J.; J. Mancheño, M.; Zamora, F. Covalent Organic Frameworks Based on SchiffBase Chemistry: Synthesis, Properties and Potential Applications. Chemical Society Reviews 2016, 45 (20), 5635-5671. https://doi.org/10.1039/C5CS00878F.

(4) Huang, N.; Wang, P.; Jiang, D. Covalent Organic Frameworks: A Materials Platform for Structural and Functional Designs. Nature Reviews Materials 2016, 1 (10), 16068. https://doi.org/10.1038/natrevmats.2016.68.

(5) Smith, B. J.; Parent, L. R.; Overholts, A. C.; Beaucage, P. A.; Bisbey, R. P.; Chavez, A. D.; Hwang, N.; Park, C.; Evans, A. M.; Gianneschi, N. C.; et al. Colloidal Covalent Organic Frameworks. ACS Cent. Sci. 2017, 3 (1), 58-65. https://doi.org/10.1021/acscentsci.6b00331.

(6) Evans, A. M.; Parent, L. R.; Flanders, N. C.; Bisbey, R. P.; Vitaku, E.; Kirschner, M. S.; Schaller, R. D.; Chen, L. X.; Gianneschi, N. C.; Dichtel, W. R. Seeded Growth of SingleCrystal Two-Dimensional Covalent Organic Frameworks. Science 2018, 361 (6397), 52-57. https://doi.org/10.1126/science.aar7883.

(7) Wang, S.; McGuirk, C. M.; d'Aquino, A.; Mason, J. A.; Mirkin, C. A. Metal-Organic Framework Nanoparticles. Advanced Materials 2018, $O$ (0), 1800202. https://doi.org/10.1002/adma.201800202.

(8) Sakata, Y.; Furukawa, S.; Kondo, M.; Hirai, K.; Horike, N.; Takashima, Y.; Uehara, H.; Louvain, N.; Meilikhov, M.; Tsuruoka, T.; et al. Shape-Memory Nanopores Induced in Coordination Frameworks by Crystal Downsizing. Science 2013, 339 (6116), 193-196. https://doi.org/10.1126/science.1231451.

(9) Sindoro, M.; Yanai, N.; Jee, A.-Y.; Granick, S. Colloidal-Sized Metal-Organic Frameworks: Synthesis and Applications. Accounts of Chemical Research 2014, 47 (2), 459-469. https://doi.org/10.1021/ar400151n.

(10) DeBlase, C. R.; Dichtel, W. R. Moving Beyond Boron: The Emergence of New Linkage Chemistries in Covalent Organic Frameworks. Macromolecules 2016, 49 (15), 5297-5305. https://doi.org/10.1021/acs.macromol.6b00891.

(11) Rodríguez- San- Miguel, D.; Yazdi, A.; Guillerm, V.; Pérez- Carvajal, J.; Puntes, V.; Maspoch, D.; Zamora, F. Confining Functional Nanoparticles into Colloidal Imine-Based COF Spheres by a Sequential Encapsulation-Crystallization Method. Chemistry - A European Journal 2017, 23 (36), 8623-8627. https://doi.org/10.1002/chem.201702072.

(12) Landis, W. J. Mineral Characterization in Calcifying Tissues: Atomic, Molecular and Macromolecular Perspectives. Connective Tissue Research 1996, 34 (4), 239-246. https://doi.org/10.3109/03008209609005267.

(13) Hildebrand. Nanoscale Control of Silica Morphology and Three-Dimensional Structure during Diatom Cell Wall Formation. Journal of Materials Research 2006, 21, 2689-2698. https://doi.org/10.1557/jmr.2006.0333.

(14) Liu, X.; Theil, E. C. Ferritins: Dynamic Management of Biological Iron and Oxygen Chemistry. Accounts of Chemical Research 2005, 38 (3), 167-175. https://doi.org/10.1021/ar0302336. 
(15) Horcajada, P.; Surblé, S.; Serre, C.; Hong, D.-Y.; Seo, Y.-K.; Chang, J.-S.; Grenèche, J.-M.; Margiolaki, I.; Férey, G. Synthesis and Catalytic Properties of MIL-100(Fe), an Iron(III) Carboxylate with Large Pores. Chemical Communications 2007, $O$ (27), 2820-2822. https://doi.org/10.1039/B704325B.

(16) Bellido, E.; Guillevic, M.; Hidalgo, T.; Santander-Ortega, M. J.; Serre, C.; Horcajada, P. Understanding the Colloidal Stability of the Mesoporous MIL-100(Fe) Nanoparticles in Physiological Media. Langmuir 2014, 30 (20), 5911-5920. https://doi.org/10.1021/la5012555.

(17) de la Peña Ruigómez, A.; Rodríguez- San- Miguel, D.; Stylianou, K. C.; Cavallini, M.; Gentili, D.; Liscio, F.; Milita, S.; Roscioni, O. M.; Ruiz- González, M. L.; Carbonell, C.; et al. Direct On-Surface Patterning of a Crystalline Laminar Covalent Organic Framework Synthesized at Room Temperature. Chemistry - A European Journal 2015, 21 (30), 1066610670. https://doi.org/10.1002/chem.201501692.

(18) Tomašić, V.; Štefanić, I.; Filipović-Vinceković, N. Adsorption, Association and Precipitation in Hexadecyltrimethylammonium Bromide/Sodium Dodecyl Sulfate Mixtures. Colloid and Polymer Science 1999, 277 (2-3), 153-163. https://doi.org/10.1007/s003960050380.

(19) Kume, G.; Gallotti, M.; Nunes, G. Review on Anionic/Cationic Surfactant Mixtures. J Surfact Deterg 2008, 11 (1), 1-11. https://doi.org/10.1007/s11743-007-1047-1.

(20) Dwars, T.; Paetzold, E.; Oehme, G. Reactions in Micellar Systems. Angewandte Chemie International Edition 2005, 44 (44), 7174-7199. https://doi.org/10.1002/anie.200501365.

(21) Kraemer, E. O.; Dexter, S. T. The Light-Scattering Capacity (Tyndall Effect) and Colloidal Behavior of Gelatine Sols and Gels. J. Phys. Chem. 1926, 31 (5), 764-782. https://doi.org/10.1021/j150275a014.

(22) Bail, A. L. Whole Powder Pattern Decomposition Methods and Applications: A Retrospection. Powder Diffraction 2005, 20 (4), 316-326. https://doi.org/10.1154/1.2135315.

(23) Petříček, V.; Dušek, M.; Palatinus, L. Crystallographic Computing System JANA2006: General Features. Zeitschrift für Kristallographie-Crystalline Materials 2014, 229 (5), 345352.

(24) Li, W.; Han, Y.-C.; Zhang, J.-L.; Wang, B.-G. Effect of Ethanol on the Aggregation Properties of Cetyltrimethylammonium Bromide Surfactant. Colloid Journal 2005, 67 (2), 159-163. https://doi.org/10.1007/s10595-005-0075-7.

(25) Patterson, A. L. The Scherrer Formula for X-Ray Particle Size Determination. Phys. Rev. 1939, 56 (10), 978-982. https://doi.org/10.1103/PhysRev.56.978.

(26) Pucci, C.; Pérez, L.; Mesa, C. L.; Pons, R. Characterization and Stability of Catanionic Vesicles Formed by Pseudo-Tetraalkyl Surfactant Mixtures. Soft Matter 2014, 10 (48), 9657-9667. https://doi.org/10.1039/C4SM01575D.

(27) Pedersen, J. S. Analysis of Small-Angle Scattering Data from Colloids and Polymer Solutions: Modeling and Least-Squares Fitting. Advances in Colloid and Interface Science 1997, 70, 171-210. http://dx.doi.org/10.1016/S0001-8686(97)00312-6.

(28) Kandambeth, S.; Dey, K.; Banerjee, R. Covalent Organic Frameworks: Chemistry beyond the Structure. Journal of the American Chemical Society 2019, 141 (5), 1807-1822. https://doi.org/10.1021/jacs.8b10334.

(29) Gao, Q.; Bai, L.; Zhang, X.; Wang, P.; Li, P.; Zeng, Y.; Zou, R.; Zhao, Y. Synthesis of Microporous Nitrogen-Rich Covalent-Organic Framework and Its Application in CO2 
Capture. Chinese Journal of Chemistry 2015, 33 (1), 90-94. https://doi.org/10.1002/cjoc.201400550.

(30) Dong, J.; Wang, Y.; Liu, G.; Cheng, Y.; Zhao, D. Isoreticular Covalent Organic Frameworks for Hydrocarbon Uptake and Separation: The Important Role of Monomer Planarity. CrystEngComm 2017, 19 (33), 4899-4904. https://doi.org/10.1039/C7CE00344G.

(31) Bai, L.; Phua, S. Z. F.; Lim, W. Q.; Jana, A.; Luo, Z.; Tham, H. P.; Zhao, L.; Gao, Q.; Zhao, Y. Nanoscale Covalent Organic Frameworks as Smart Carriers for Drug Delivery. Chem. Commun. 2016, 52 (22), 4128-4131. https://doi.org/10.1039/C6CC00853D.

(32) Horcajada, P.; Chalati, T.; Serre, C.; Gillet, B.; Sebrie, C.; Baati, T.; Eubank, J. F.; Heurtaux, D.; Clayette, P.; Kreuz, C.; et al. Porous Metal-Organic-Framework Nanoscale Carriers as a Potential Platform for Drug Delivery and Imaging. Nature Materials 2010, 9 (2), 172-178. https://doi.org/10.1038/nmat2608.

Acknowledgments: We acknowledge DESY (Hamburg, Germany), a member of the Helmholtz Association HGF, for the provision of experimental facilities. Synchrotron X-ray diffraction experiments with COF-TAPB-BTCA were carried out at the beamline P02.1 PETRA III under the proposal I-20170717 EC.

Funding: This work was supported by the European Union (ERC-2015-STG microCrysFact 677020), the Swiss National Science Foundation (Project no. 200021_181988), ETH Zürich and Ministry of Science, Innovation and Universities MICINN (MAT2016-77608-C3-1P). A.E.P.P. acknowledges a TALENTO grant (2017-T1/IND5148) from Comunidad de Madrid. D.M. acknowledges financial support from the European Union (ERC-Co 615954). ICN2 is supported by the Severo Ochoa program from the Spanish MINECO (Grant No. SEV-2017-0706).

Competing interests: A patent related to the work presented in this document has been filed.

Data and materials availability: All data needed to evaluate the conclusions in the article is present in the main text and supplementary materials.

Supplementary Materials: Materials and Methods; Supplementary Text; Figures S1-S31;

Tables S1; Movie S1. 


\section{Table of Contents}
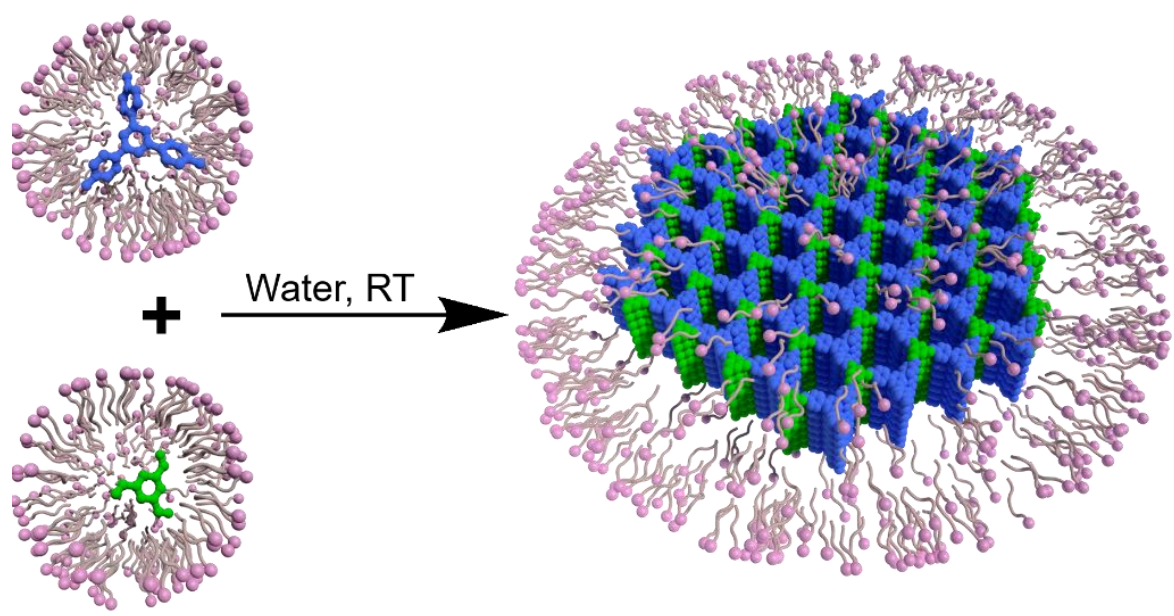

444 